\title{
SYPHILIS OF THE HEART AND AORTA (WITH SPECIAL REFERENCE TO THE EARLY STAGE OF THE DISEASE) AND ITS APPROPRIATE TREATMENT *
}

By SIR THOMAS HORDER, Bart., M.D., F.R.C.P.

Mr. President, Ladies and Gentlemen :

I do not think I need to stay to justify the suggestion which I made to our worthy Secretary a little while ago, that it might be appropriate if this Society were to discuss the particular subject which has been advertised, namely, "Syphilis of the Heart and Aorta (with special reference to the early stage of the disease) and its appropriate treatment."

Clearly, this subject is one of great importance; the fact that we know very little about it does not, of course, excuse our not discussing the matter, but rather makes it more necessary that we shouid.

It seems to me that there are four basic facts which render this subject one of supreme importance. The first is that, as we all believe, syphilis is a disease which is eminently amenable to treatment. The second basic fact is that the success of such treatment in this disease process, as in so many others, is directly proportionate to the early stage at which the diagnosis is made and the treatment begun. The third fact is that the tissues concerned are of vital importance, not only to health, but to life. And the fourth basic fact is, that if once structural changes are produced before the presence of the disease is recognised, then, even though healing takes place-as we know it does to a large extent - the secondary changes produced in the heart and in the aorta are of great importance in leading to limitation of activities and even to premature death. It behoves us therefore to make a big effort to diagnose and treat the disease before these residual changes in structure take place. It might be said that syphilis of these organs is a rare condition, why, therefore, bother

* Based upon an address delivered before the Medica! Society for the Study of Venereal Diseases on February 26th, 1926. 


\section{BRITISH JOURNAL OF VENEREAL DISEASES}

very much about it? It so happens that we get ample evidence, in the post-mortem room, that that statement is not correct. There is this incongruity between careful post-mortem observation and clinical experience: that whereas in clinical work we rather seldom satisfy ourselves that we really are dealing with an early case of syphilitic disease of the aorta or of the heart, in the post-mortem room we are frequently convinced that the changes we meet with are primarily of syphilitic origin. This incongruity makes it imperative, I think, that we should constantly bear in mind the prevalence of the disease, and that we should be constantly on the look-out for its occurrence. It is prone to be very latent; that is the inference which one must inevitably draw from the discrepancy between post-mortem and clinical experience. The latency of the disease causes it to elude us until such changes have been produced which I referred to as being structural and permanent. I suppose one of the chief reasons why the disease is so latent is, that neither of the two structures of the heart which yield us definite physical signs is often affected by syphilis ; I refer to the pericardium and the endocardium. Syphilitic pericarditis must be almost a curiosity; and syphilitic endocarditis, in the sense I am referring to syphilitic disease to-night, namely, in an early and curable stage, is, again, hardly a recognised clinical entity. We are all familiar with cases of aortic incompetence which we have plenty of reason to attribute to old syphilis, and I sometimes draw attention to what I believe to be a parasyphilitic lesion of the mitral cusps, taking the form of mitral regurgitation, with a tendency to a highly musical quality in the apical systolic bruit, the sort of thing one comes across casually during routine examination in cases of tabes dorsalis. Again, we know how typical a condition a state of heart-block may be where there has been produced definite anatomical change in the bundle of His. And, thirdly, perhaps most significant of all, there are the signs and symptoms of aortic aneurysm, though the nature of these signs and symptoms goes rather too often unrevealed if we do not constantly remember the possibility of its existence; still, the signs and symptoms of aneurysm of the thoracic aorta are sufficiently standardised to yield good criteria for diagnosis.

I do not propose to deal with these three groups of what 


\section{SYPHILIS OF THE HEART AND AORTA}

I may call parasyphilitic disease of the cardio-vascular system; I want specially to draw attention to the disease in its early and progressive stages, to consider what are its manifestations, and, after that, to consider briefly what is the appropriate treatment.

There seems very little doubt that the thoracic aorta is more frequently attacked by syphilis in these early stages than is the heart itself, if we may judge-and this is the only criterion by which we can judge of the incidence of the disease-by the post-mortem data. General considerations lead us to believe that the first, or ascending, part of the thoracic arch is the zone of attack par excellence. I suppose we all take the view that there are two specially liable zones in the arterial system for syphilitic attack; one is the circle of Willis and its branches, and the other is the base of the aorta and the part immediately above it. We have, I think, ample reason for believing that there is about the same duration of time elapsing between primary infection and manifestations of syphilitic aortitis, as there is between primary infection and manifestations of socalled cerebral syphilis (syphilitic arteritis involving one or more branches of the circle of Willis), that is to say, something between five and ten years. I shall stress this point, because I think there is a general feeling-which I do not share-that the disease, when it occurs, is later in the course of the life-history of syphilitic processes than I have mentioned. So it is, for preference, the man-or woman, but chiefly the man-of twenty-five to thirty-five in whom one must suspect early aortitis of a syphilitic kind. I say I do not think there is any difference between the age of incidence in the two common zones of attack. And we know that the type of patient who develops symptoms of cerebral arteritis (endarteritis obliterans), whether by epileptiform attacks or transient diplopias or mono- or hemi-plegia, or whatever the manifestation may be, is generally in the decade 25 to 35 . All sorts of developments later in life occur; aneurysm later, aortic regurgitation later. But, as I have already mentionèd, we want to be earlier on the scene than when those later, more residual, processes are what bring the patient to us.

What are the symptoms of this early condition? Let us take the aorta first. They are largely problematical, and this is the main reason why I have brought the subject forward, because I want to know if any of us are 


\section{BRITISH JOURNAL OF VENERFAL DISEASES}

capable of making contributions which will stand the test of experience. I shall mention the things to which I am prone to attach great importance, but I shall not say they take me past the point of suspecting that the patient may be developing, or may have developed, syphilitic aortitis, because I shall admit I am never quite certain of the diagnosis, though I am certain, or may be certain, of the wisdom of treating the patient as a suspect. The kind of thing is this: I attach great importance to substernal pain, which I can reasonably attribute to the aorta, in a young or middle-aged man who has no other adequate reason why he should have pain of an anginoid type in the sternal region. Like all other anginoid pains, it is prone to be increased by effort, but by no means necessarily induced by effort. It may be induced by other things to which knowledgable patients are apt to attribute the pain, and if we are not on the alert we may take their explanation as sufficient. One of the cases I thought most certainly had this disease was sent to me as a case of rather intractable and severe cardiospasm. The pain was attributed to acid dyspepsia in a nervous man. But his pain, I thought, on investigating it fully, was definitely aortic pain. I was not quite satisfied with his aortic second sound-I shall refer to that under physical signs - he gave a clear history of infection seven years before ; his blood gave a trebly-plus Wassermann, and the Sigma reaction was very definite. And if one may judge by therapeusis-but that argument is very fallible, inasmuch as the case was one in which recumbent rest was indicated-treatment on anti-syphilitic lines with prolonged rest cured him of the pain. I think pain, in the subjective group of data, is most important.

Secondly, there are other subjective features, less helpful because more vague. A sense of oppression, of discomfort, in the chest, increased by effort ; in short, almost an " aortic consciousness" rather than a " heart consciousness." Thirdly, shortness of breath-but now I am getting still more vague in the symptomatology, seeing that the causes of shortness of breath are so numerous-a feeling of slackness, and finding the patient a little anæmic.

Turning now to the physical signs, I have already said that I shall exclude conditions in which physical signs indicate valvular disease, aneurysm or some such condi- 


\section{SYPHILIS OF THE HEART AND AORTA}

tion as a serious myocardial lesion. The physical signs are entirely problematical, and I submit them to you for consideration and for further suggestion. I have never found one of these patients in whom I could satisfy myself that the aorta showed signs of dilatation. $\mathrm{l}$ know it is described ; French physicians construct a clinical picture of aortitis which is more elaborate than ours, they speak of a definitely widened aortic shadow, they even speak of increased width of the parasternal dulness to percussion. I have never satisfied myself that I can get that picture. After all, why should such a picture be presented? Syphilitic mes-aortitis- to give it its more accurate term -is a patchy condition, and if it were so diffuse as to lead to dilatation of the aorta in a man of thirty, or even forty, it would obviously be a very serious condition indeed. The patulous aorta of an older patient, or the dilated aorta of a highly atheromatous subject, we are familiar with. But in the type of case I have in my mind I cannot say I have satisfied myself about an increase in the size of the aorta by any of the methods we are familiar with. But coming to the character of the heart sounds, I think there is something which is perhaps helpful. The aortic second sound should, in all cases of substernal pain, be criticised very carefully indeed. There are two features that I should like discussion upon. The first is as to whether or not a little blurring of the aortic second sound, which is not a definite bruit, so that one cannot say there is an aortic diastolic bruit, but merely that the aortic second sound is not clear-and we should remember that the aortic second sound is the clearest of all the heart soundswhether a little blurring should not attract our attention seriously. And I would like to say here that I attach great importance to listening to the aortic second sound, not merely over the anatomical point of the valve-the junction of the second right costal interspace with the sternum-but down the sternum and at the bottom of the sternum. The late Sir William Osler spoke of the pulmonary base as being the " area of auscultatory romance," a very attractive label to give it. I have ventured sometimes to call the region of the xiphoid the " area of auscultatory neglect," and I think that label is justified by what one finds not infrequently, namely, a fairly wellmarked, definite but distant, aortic bruit, there but nowhere else. Given a quiet room, and given that one's 


\section{BRITISH JOURNAL OF VENEREAL DISEASES}

attention is directed definitely to any abnormality in sound, then I think that sometimes one does not pass an aortic second sound heard in that situation, whereas one may pass it when heard elsewhere.

My second point with regard to the aortic second sound is what might strike some as being rather the opposite condition, namely, accentuation in the absence of hyperpiesis. To me, the significance of that is problematical ; I do not attach so much importance to it as I do to a blurring of the second sound.

I know no other symptoms and signs than these which I can, from experience, correlate with early syphilitic disease of the aorta. Of course, collateral signs of syphilis are very valuable, and it is worth remembering that we do not always see these unless there is in our mind the possibility of syphilitic disease : leucoplakia, old scars, pigmentations, and so on. Collateral evidence of syphilis, visceral or otherwise, would be an important matter in a case of this kind. Lastly, any suspicious case is naturally subjected to the complement-fixation test. I have already said that, once aortic incompetence has developed, the case is outside the category of those I was anxious we should discuss to-night. I do not regard the mere presence of any aortic diastolic bruit as proof of permanent aortic incompetence. I do not know if any one who is here to-night has had experience of a patient who had quite a definite, well-marked aortic diastolic bruit and has heard it disappear with treatment, but I have once met with that experience. Fortunately for my own conviction, the case was examined by another physician, and we both agreed that this patient had this well-marked aortic diastolic bruit. It was not only heard at the bottom of the sternum, but down the left side of the sternum and at the aortic base. The patient was a soldier home from India on leave, and, seeing that he had a good deal of pain of an anginoid character and gave no history of rheumatic fever or other likely cause of aortic regurgitation, his blood was examined, with a positive result. He was put under intensive anti-syphilitic treatment. Four months after the treatment was begun his aortic second sound was clear. I was rather surprised to find it so. I watched him for some weeks, and eventually took him to my colleague who had examined him with me, and he agreed that the second sound was clear. It is the only 


\section{SYPHILIS OF THE HEART AND AORTA}

case in which I have known a well-marked, indubitable aortic bruit clear up under treatment. I refer to that because, just now, I spoke of aortic valve lesions and said they were residual changes, but I was then referring to cases which had permanent valvular defect.

Now as to the heart. We can diagnose the condition here less commonly, I think, because it is more latent than in the aorta, probably because we are more often denied the evidence which I think of the greatest importance in early diagnosis of aortic syphilis, namely, pain. It is uncommon in syphilitic disease of the heart, so far as I am aware. Sudden death by rupture of an aneurysm in the heart wall, or by the production of an ischæmic infarct the result of endarteritis of branches of the coronary vessels, is not uncommon; but those happenings are outside the range both of diagnosis and of therapeutics.

What symptoms and signs have we got in connection with the heart which are at all suggestive? I only know one, and that is heart-block, or the so-called StokesAdams syndrome, to which I have already referred. If any degree of that syndrome is observed in a man under fifty, there is always the hope that his syphilitic disease is, even if not early, still amenable to treatment. If he is under forty, the chance is still better. Over sixty, we know that the group of cases of heart-block is not usually syphilitic in this sense of active syphilis, though syphilis may have been an ætiological factor years ago ; but by this time there is degeneration of so serious a form that the condition does not respond to anti-syphilitic remedies.

Before I speak of treatment, I would like to refer to one case which is so unusual that it deserves mention. All these lesions, I have already said, are apt to be latent and to escape us. A rare event may bring the patient to us, as in this case. A police constable, aged thirty-two, who was helping a colleague to take a dead body down some awkward and dark stairs (the patient being the top man and having the head and shoulders of the body), had his balance upset by his colleague stumbling, and in the effort to prevent his burden from following down the stairs, produced what I thought was a rupture of the aortic cusp. There was sudden severe pain, so that the constable let go his burden. The pain moderated in a few seconds, leaving a dull ache, which he referred to the region of the manubrium: This persisted, and he felt 


\section{BRITISH JOURNAL OF VENEREAL DISEASES}

somewhat dizzy; when he got outside he was faint. He sat down for a little while, found he was not better, and so reported sick and went to bed. His wife, who was out at the time, went to see him and make him comfortable, and then began to look under the bed. The man asked her what she was looking for, and she said, "The cat is up here, I can hear it purring." When he was seen by the police surgeon there was a loud cardiac bruit, quite evident before the heart was listened to at all ; when standing at the foot of the bed the bruit was easily heard as a purr. Realising that something unusual had occurred, the man was put on an ambulance and sent to St. Bartholomew's Hospital; that was ten days later. When he arrived there he still had a bruit which could be heard some distance off. He was anæmic; he had no signs of enlargement of his heart at that time. He had a little substernal discomfort, and a trebly-positive Wassermann. He was put under treatment and got well, except that he left the hospital with a well-marked aortic diastolic bruit. No doubt that was a case of syphilitic disease of the aorta with some softening of the aortic cusps, or at least one cusp, which was brought to light by the extraordinary accident which produced a rupture or split of the diseased cusp.

In the matter of treatment I have nòt much to say. I am often asked whether, when it is once decided to treat a case of some vascular lesion of supposed syphilitic nature, we should go slowly with anti-syphilitic remedies or proceed as in other types of the disease. I think, as a matter of principle, we should proceed rather cautiously, but I am not sure that there is any need to be very slow. But there is great need to proceed as intensively as possible consistent with not getting a disaster ; I cannot say I have seen a disaster. I have seen neo-kharsivan and other derivatives given with great boldness intravenously to cases of arterial disease other than syphilitic ones, and I have trembled a little for the result. There was a vogue for treating cases of ulcerative endocarditis by that method, and I remember a colleague of mine who had on a previous occasion discussed the question of how cautious one should be in treating a patient with an aortic aneurysm with neo-kharsivan who did not hesitate about his doses in endocarditis. My practice is to be very intensive and very prompt with mercury in these cases, 


\section{SYPHILIS OF THE HEART AND AORTA}

giving iodide at the same time, then to follow with a routine series of doses of novarsenobillon or neo-kharsivan. When I have once subjected a patient to a course of mercury and iodide, I do not give any smaller doses of the arsenobenzol than I do in other syphilitic diseases. But I ought to have first stressed one point very strongly, and that is that I think very prolonged recumbent rest is indicated. This must be insisted upon, and the term is in months rather than in weeks. 